\title{
Experimental investigation of the boundary layer transition on the prismatic blade using infrared thermography
}

\author{
Erik Flídr $^{1, *}$, and Tomáš Jelínek ${ }^{1}$ \\ ${ }^{1}$ VZLU - Czech Aerospace Research Centre, Beranových 130, 19905 Prague, Czech Republic
}

\begin{abstract}
Topic of this contribution is the detection of the laminarturbulent boundary layer transition on the prismatic blade using infrared thermography. Several Reynolds and Mach numbers were investigated, and their influence on the boundary layer transition was evaluated. Results are in good agreement with experiments that were performed earlier with hot-film anemometry.
\end{abstract}

\section{Introduction}

The kinetic energy loss of the flow caused by the presence of the turbomachinery blades in subsonic flow regimes is strongly dependent on the character of the boundary layer (BL) on the blades and also on the separation of the BL before the trailing edge. The character of $\mathrm{BL}$ is dependent on the flow conditions which are commonly described by similarity criteria such as Mach and Reynolds numbers. Laminar-turbulent transition of the BL is also influenced by the turbulence level of the free stream, by the pressure gradients, and by the compressibility effect, see Narasimha [1] or Mayle [2]. The kinetic energy loss can be determined experimentally by the pressure measurement behind the trailing edges of the blades. Nowadays the Computational Fluid Dynamics (CFD) is widely used in the turbomachinery design and analysis of the flow field. Fully turbulent computations are used very often and the results are satisfying for high Reynolds number regimes (higher than $10^{6}$ ), where the transition of the BL starts very early behind the leading edge.

For low Reynolds number (lower than $10^{6}$ ) flow the BL transition must be taken into account if meaningful results of the CFD computations are to be obtained. Therefore several BL transition models were developed and are widely tested in various examples by Straka et al. [3, 4], Menter et al. [5], Suzen and Huang [6] or Steelant and Dick [7]. To validate the CFD models it is necessary to have the relevant experimental data of the $\mathrm{BL}$ transition for the different flow conditions.

BL transition can be investigated using various experimental methods, e.g. surface hotfilm anemometry (HFA) or infrared thermography. Many other methods can be found in literature, see e.g. Tropea et al. [8].

Gomes et al. [9] investigated BL transition using HFA. They used an array with 50 sensors for the detection of BL transition. Measurements were performed in the constant

* Corresponding author: flidr@,vzlu.cz 
temperature anemometry (CTA) regime. Comparison of HFA and hot-wire anemometry (HWA) measurement at the blade cascade was made by Griffin and Davis [10].

Infrared thermography uses differences in the temperature of the body surface to detect the BL transition. These differences are caused by the variation of the heat transfer that is associated with the BL type. Across the turbulent BL, there is higher heat transfer due to the enhanced momentum transfer. Therefore, the body surface will be more heated or cooled, depending on whether the body has lower or higher temperature compared to the oncoming flow. The major advantage of this method compared to the other methods used for the detection of the BL transition is, that the flow field is not influenced by any objects on the surface (e.g. presence of the HFA in the flow field). That is very important, because of a high sensitivity of the BL transition to any disturbances. Howerver, it is only qualitative method how to detect the BL transition and parameters of the flow cannot be determined.

The key elements to obtain meaningful results from the experiment are the differences in temperature between the flow and the body surface and low thermal conductivity of the model body.

Because of reasons mentioned above, many authors use this technique for the detection of the BL transition. This technique was used in the supersonic flow by Saric and Zuccher [11], where for the better results, surface of the model was cooled by the liquid nitrogen or heated by the electrical element during some measurements. Model was also cooled by Yokokava [12], who used cooling blankets for the surface temperature decreasing and experiments were performed in low speed wind tunnel. An electric element for the model heating was used by Liselle at al. [13], De Luca et al. [14] and Patorski et al. [15]. Another technique for the surface heating is to use heat lamp as was shown by Baek and Fuglsang [16] or Raffel et al. [17].

As the initial experiment, the transition on single laminar airfoil at the ambient temperature in the intermittent suck-down wind tunnel was performed at our laboratory by Jelínek [18]. The experiment was designed to study the transition evoked by different thickness of turbulence-generators.

In closed-loop wind tunnels the difference in temperature of the flow and the body surface is an issue. As the infrared cameras can detect roughly $30 \times 10^{-3}{ }^{\circ} \mathrm{C}$, the difference of surface temperature of $1{ }^{\circ} \mathrm{C}$ seems to be satisfactory to detect the transition. Otherwise the temperature of the model or the flow must be changed by external source. Both approaches are presented and discussed in this paper.

\section{Experimental methods, setup and apparatus}

\subsection{Wind tunnel and flow regime}

Experiments were performed in VZLU laboratory of high speed aerodynamics in a closed loop low-pressure wind tunnel (WT). Scheme of the WT is shown in Figure 1.

A linear blade cascade that was assembled of the individual prismatic blades placed between the two side-walls made of plexiglass. Pitch-to-chord ratio of the cascade was $t / c=0.9$. The flow incidence could be adjusted, with respect to the fixed cascade, by a pair of shaped semi-nozzles placed upstream of the linear cascade. Inlet flow angle was set at constant value $\alpha=85^{\circ}$. Configuration of the wind tunnel allows adjusting the Reynolds and Mach number independently. Mach number can be changed by changing the rotationalspeed of the compressor, while the change of the Reynolds number can be achieved by reduction of the pressure in the WT by set of vacuum pumps.

Flow regimes with tunnel settings are summarized in Table 1. 
Regime of the flow in the WT was set based on pressure measurement. The reference total pressure was measured in the inlet chamber and the static pressure at the wall in front of the cascade. All pressures were converted to the voltage output using pressure transducers with accuracy of $0.1 \% \mathrm{rdg}$. Barometric pressure was measured by means of Druck DPI 145 with accuracy of $0.013 \%$ FS. The measured signals were sampled using A/D card (National Instruments PCI-6259 A/D card: 16-Bit, 1MS/s (Multichannel), 1.25 MS/s (1-Channel), 32 Analogue Inputs). The rate could be set according to frequencies of the observed process. Signal conditioning was provided by DEWETRON system, which was equipped with modules with bandwidth up to $200 \mathrm{kHz}$. Temperature and humidity of the flow was measured using hygrometer Sensorika Humistar HTP-1.

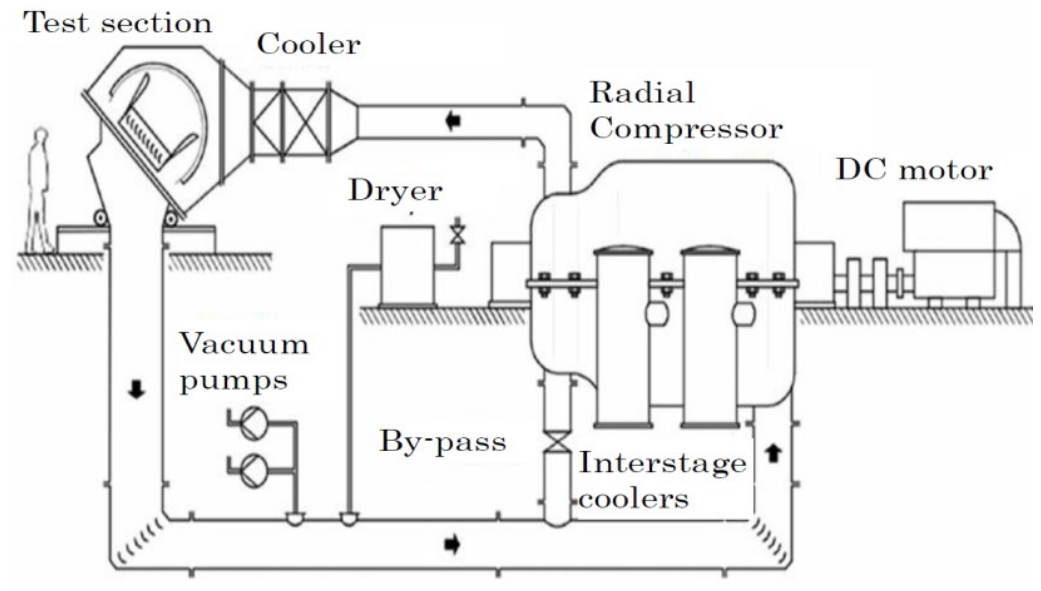

Fig. 1. Scheme of the wind tunnel.

According to the measurement device accuracy, uncertainties of the measured quantities were established according to the GUM document [19]. For the outlet isentropic Mach number $M_{2 i s}$, the uncertainty was less than $1 \%$.

Table 1. Tested flow regimes.

\begin{tabular}{|c|c|c|c|c|c|c|c|c|}
\hline \multicolumn{4}{|c|}{ Blade made of Cibatool - blade $A$} & \multicolumn{5}{|c|}{$\begin{array}{l}\text { Blade made of PLA natural by 3D printing - } \\
\text { blade B }\end{array}$} \\
\hline $\begin{array}{c}\mathrm{M}_{2 \text { is }} \\
{[1]}\end{array}$ & $\begin{array}{c}\operatorname{Re}_{2 \text { is }} \\
{[1]}\end{array}$ & $\begin{array}{r}\mathrm{T}_{\text {sref }} \\
{\left[{ }^{\circ} \mathrm{C}\right]}\end{array}$ & $\begin{array}{c}\mathrm{T}_{\mathrm{a}} \\
{\left[{ }^{\circ} \mathrm{C}\right]}\end{array}$ & $\begin{array}{c}\mathrm{M}_{2 \text { is }} \\
{[1]}\end{array}$ & $\begin{array}{c}\operatorname{Re}_{2 \text { is }} \\
{[1]}\end{array}$ & $\begin{array}{l}\mathrm{T}_{\text {sref }} \\
{\left[{ }^{\circ} \mathrm{C}\right]}\end{array}$ & $\begin{array}{c}\mathrm{T}_{\mathrm{a}} \\
{\left[{ }^{\circ} \mathrm{C}\right]}\end{array}$ & \\
\hline 0.8 & $5.0 \times 10^{5}$ & 12.7 & 14.1 & 0.8 & $5.0 \times 10^{5}$ & 15.7 & 17.7 & \multirow[t]{6}{*}{ Unheated } \\
\hline 0.6 & $7.5 \times 10^{5}$ & 12.7 & 13.5 & 0.6 & $7.5 \times 10^{5}$ & 15.5 & 16.5 & \\
\hline 0.6 & $5.0 \times 10^{5}$ & 13.2 & 14.0 & 0.6 & $5.0 \times 10^{5}$ & 16.0 & 17.0 & \\
\hline 0.6 & $2.5 \times 10^{5}$ & 13.1 & 14.0 & 0.6 & $2.5 \times 10^{5}$ & 15.4 & 16.1 & \\
\hline 0.4 & $5.0 \times 10^{5}$ & 12.7 & 13.3 & 0.4 & $5.0 \times 10^{5}$ & 16.0 & 16.6 & \\
\hline 0.4 & $2.5 \times 10^{5}$ & 16.1 & 16.5 & 0.4 & $2.5 \times 10^{5}$ & 19.5 & 19.7 & \\
\hline- & - & - & - & 0.4 & $2.5 \times 10^{5}$ & 22.3 & 20.1 & Heated \\
\hline- & - & - & - & 0.4 & $5.0 \times 10^{5}$ & 24.2 & 21.7 & \\
\hline
\end{tabular}

\subsection{Infrared camera}

\subsubsection{Principle of the Infrared thermography}

Infrared thermography (IRT) is a method for direct measurement of the emissive power of the body surface. Relation between the temperature and emissive power of the body is given by Stefan-Boltzmann law: 


$$
E=\varepsilon \sigma T^{4},
$$

where $\varepsilon$ is the emissivity of the body ( $\varepsilon=1$ is valid for black body), $\sigma=5.68 \times 10^{-8} \mathrm{~W} / \mathrm{m}^{2} \mathrm{~K}^{4}$ is the Stefan-Boltzmann constant and $T$ is the thermodynamics temperature. Proper camera calibration and the knowledge of the body emissivity is essential for the correct temperature measurements. Here is important to note that the emissivity $\varepsilon$ is the function of four variables and can be expressed as function of:

$$
\varepsilon=f(\eta, T, \varphi, R),
$$

where $\eta$ is the spectral range, $\varphi$ is the direction of radiation and $R$ is the roughness of the surface. Because of experimental setup, $R$ and $\eta$ can be neglected (the blade surface was smooth and spectral range $\eta=7.5 \div 14 \mu \mathrm{m}$ ), but the $\varphi$ influenced the results as will be shown further below in section 3 .

There is one more issue with IRT, the reflection from the body surface. This phenomenon will be also discussed in the section 3 .

\subsubsection{Infrared camera setup}

FLIR A655sc infrared camera with resolution of $640 \times 480$ pixels and maximum frame rate of $50 \mathrm{~Hz}$ was used for the experiments. View angle of the camera was $15^{\circ}$. Camera can clearly detect $30 \times 10^{-3}{ }^{\circ} \mathrm{C}$ differences in temperature. Spectral range of the camera is $\eta=$ $7.5 \div 14 \mu \mathrm{m}$. Standard temperature range for the FLIR A655sc is $T=(-40 \div 150){ }^{\circ} \mathrm{C}$ and can operate in the temperature range of $T=(-10 \div 50){ }^{\circ} \mathrm{C}$. Best accuracy of the camera is $\pm 2{ }^{\circ} \mathrm{C}$ or $\pm 2 \% \mathrm{rdg}$.

Camera was placed $260 \mathrm{~mm}$ behind the trailing edge of the blades, see Figure 2.

\subsection{Hot film anemometry}

To identify the transition in the boundary layer the array of hot film sensors was used at first. The sensors were manufactured by Tao systems and were delivered as a set on one polyamide foil with a thickness of about $0.05 \mu \mathrm{m}$. The foil was glued to the blade suction side, with asymmetrical distribution of the sensors around the blade centreline (see Figure 3). The sensors were made of two copper leads and the active part was made from nickel (thickness $0.2 \mu \mathrm{m}$ ). Therefore, it was necessary to minimize the thermal conductivity between sensors and the blade, the blade was made of Cibatool (further

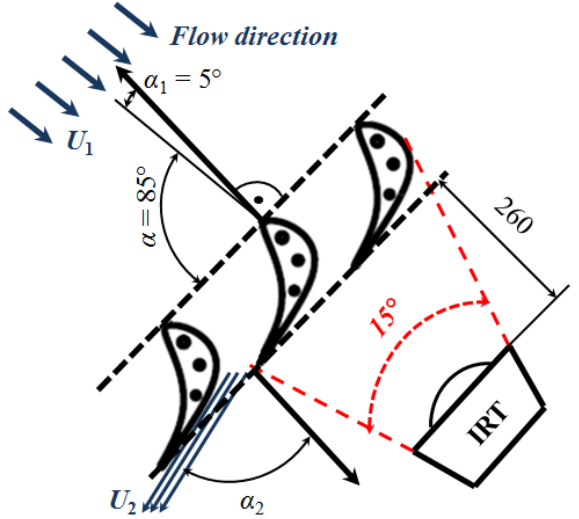

Fig. 2. Blade cascade with IR camera.

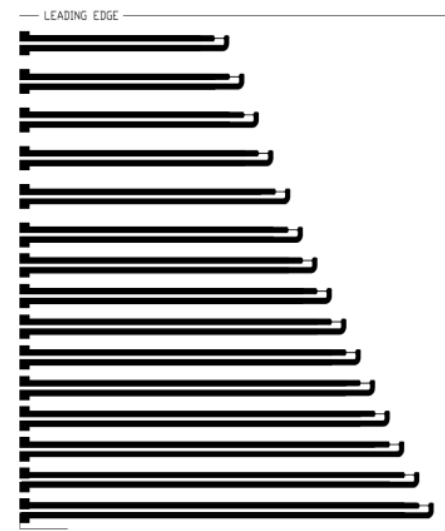

Fig. 3. HF sensors positions. 
referred to as blade $\mathrm{A})$.

Constant current mode was used for the measurements. Hot-films signals were acquired for 5 seconds with sampling frequency of $f_{\text {samp. }}=50 \mathrm{kHz}$ and with low pass filter of $f_{\mathrm{LP}}=10 \mathrm{kHz}$. Results from these experiments were summarized in Michálek [20].

\subsection{Blades for the visualization of boundary layer transition}

Two different blades were used for visualization experiments using IR camera. Prior to use of blade A (made of Cibatool), the hot film sensors were removed and the surface cleaned. A new blade (further referred to as blade B) was manufactured (made of PLA) by the 3D printer in the VZLU laboratory of high speed aerodynamics. The blade was equipped with the cavity and with a thin slit, leading from the cavity to the trailing edge, see Figure $4 \mathrm{a}$. The copper plate was placed to the slit for better heat transfer trough the blade. For better resolution of the camera, blade was heated using hot air. Hot air was blown into the cavity through hole on one side of the blade and was sucked out on the other.

To determine the position of the BL transition detected by IR camera it was necessary to implement another coordinates. Usually there are two coordinates systems for the description of the location on the blade surface. Orthogonal system $(x, y)$ and curvilinear system with coordinate $s$ (see Figure $4 \mathrm{~b}$ ), that is associated with blade surface. However IR camera was rotated by angle $\varphi=45^{\circ}$ against system $(x, y)$ and was rotated by angle $\varepsilon=$ $15^{\circ}$ against tangent $t$, which leads through the tangent point in trailing edge, see Figure $4 \mathrm{~b}$.

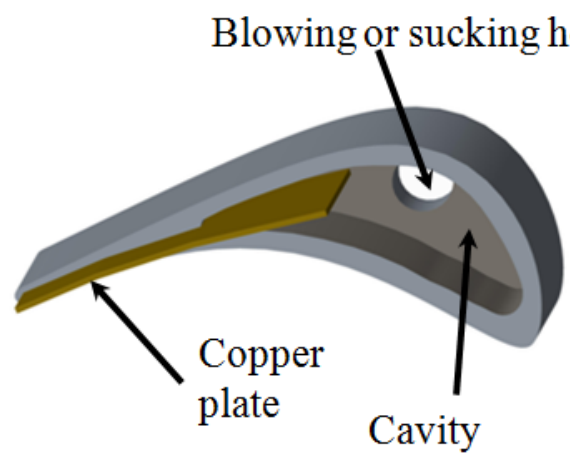

a)

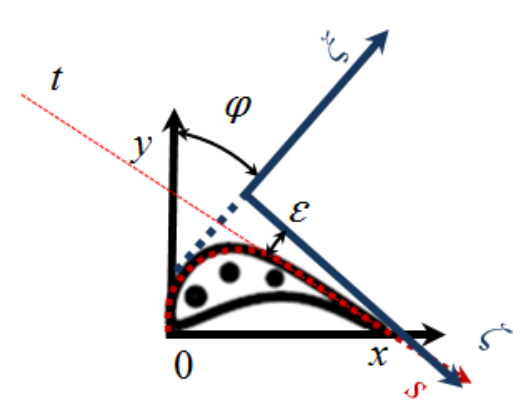

b)

Fig. 4. a) Blade made by $3 \mathrm{D}$ print, b) coordinate systems.

\subsection{Detection of the boundary layer transition}

As was mentioned above in section 1, a heat transfer increases significantly when laminar to turbulent BL transition occurs. This is caused by the increase of skin fiction coefficient $C_{\mathrm{f}}=\tau_{\mathrm{w}} /\left(2 \rho u^{2}\right)$, that is usually one order higher in the turbulent BL compared to the laminar BL. According to Reynolds analogy, heat transfer is a function of skin friction coefficient as:

$$
N u=1 / 2 C_{\mathrm{f}}(x) R e,
$$

where $N u=h l / \lambda$ is the Nusselt number and $R e=u l / v$ is the Reynolds number. Nomenclature for the Nusselt and Reynolds number is: $h$ is the heat transfer coefficient, $l$ is the characteristic length, $\lambda$ is the thermal conductivity of the fluid, $u$ is the fluid velocity and $v$ is the kinematic viscosity. Experiments were performed with air as a medium, therefore the 
Prandtl number was approximately 1 and the condition for using Reynolds analogy was satisfied.

\section{Results and discussion}

\subsection{Hot film anemometry}

Root mean square (RMS) values of the measured voltages signals from hot-films are shown in Figure 5. Results show, that the BL transition occurs near the trailing edge of the blade $(s / c>0.8)$ where the RMS values of the signals increase for all measured regimes of flow. Free stream turbulence during these measurements was under $2 \%$, as was shown by Michálek [20].

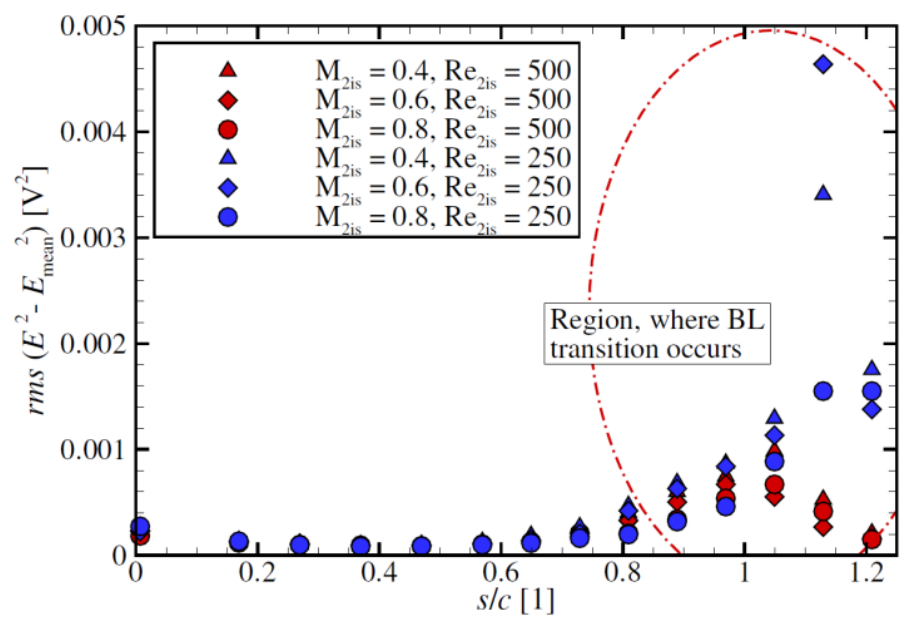

Fig. 5. RMS values of the measured voltage signals from the HFA.

\subsection{Effects that influence the IRT measurement and initial conditions}

During the initial testing, blades A and Bwere placed in the test section at the same time. This configuration was found to be unsatisfactory for the detection of BL transition; however the images can be used for the demonstration of the artefacts that were observed during the IRT measurement. The images in Figure 6 were captured before the WT was turned on.

Blade B was placed above the blade A. The blade B was heated at the temperature $T=26{ }^{\circ} \mathrm{C}$, while the blade A had temperature of the surroundings that was $T_{\mathrm{a}}=11^{\circ} \mathrm{C}$.

Regions, where hot air was blown in the blade B and sucked out (places with higher temperature close to the leading edge), can be seen in Figure 6. At the leading edge of the blade there is also the region where the influence of the curved shape can be observed. This conclusion was made because there were no other reasons for such significant decrease of temperature. At the trailing edge of the blade in the upper image in Figure 6 there can be seen a region with the significant decrease in temperature, it is a part of the blade, where the copper plate did not reach. 


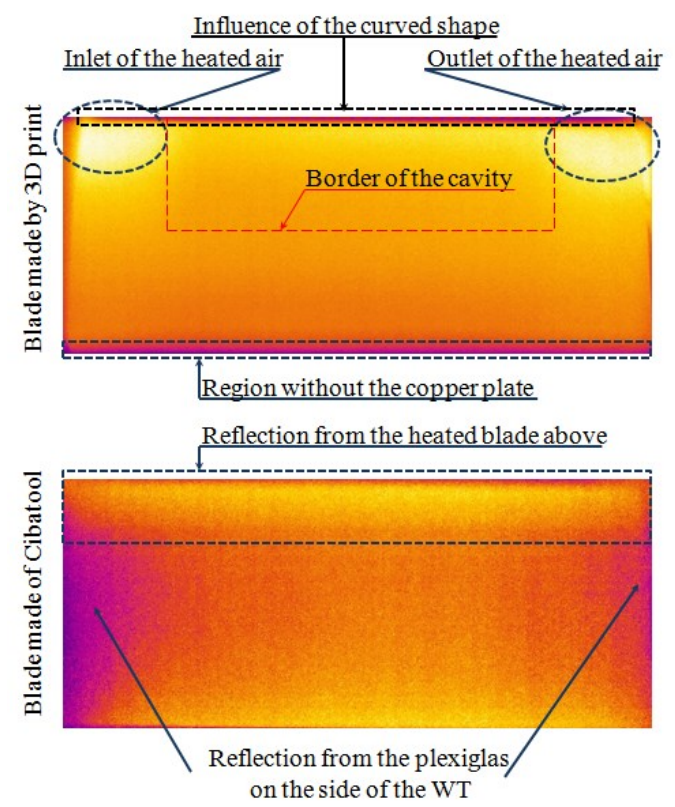

Fig. 6. Effects influences the IRT measurement.

The IRT image of blade A is shown in the lower part of the Figure 6. Region with the reflected radiations, which influence the IRT temperature reading, from both plexiglasses in which the cascade was assembled, are visible. Reflection of the radiation from the heated blade can be seen behind the leading edge in Figure 6 . Reflection ends at the point, where the step change of the temperature occurs.

\subsection{Boundary layer transition}

\subsubsection{Transition on blade $A$ and unheated blade $B$}

The BL transition was investigated for several flow regimes described in Table 1.

All regimes were measured with the blade A first and after the measurement was finished the blade A was replaced with the blade B.

Visualization of the BL transition is shown in Figure 7. Surface temperature distribution on the blade centreline is shown on the right hand side of Figure 7. Ratios of the temperatures at each point along the centreline and maximum temperature $T_{\mathrm{s}} / T_{\max }$ are plotted in the figure. Mean value from the surface temperature distribution was chosen as a reference surface temperature $T_{\text {sref. }}$ Free stream temperature $T_{\mathrm{a}}$ was higher compared to blade surface temperature $T_{\text {sref }}$ (see Table ) during all tests.

Corner vortices can be seen in every picture in Figure 7. On the right handside of figure, vorticity is enhanced due to the leakage of the fluid through the gap between the blade and the sidewall that was created during the cascade mounting. Because the temperature of the plexiglases was lower than the temperature of the free stream, air that goes through these gaps on both sides of the WT was cooled and as a result of this phenomenon the blades in the region of corner vortex were cooled as well.

Visualization of the BL transition using IRT technique shows good agreement in the location of the transition on the blade A and B for all tested regimes. The transition occurs near the trailing edges of the blade for the $\operatorname{Re}_{2 \text { is }}=2.5 \times 10^{5}$ and for both $M=0.4$ and 0.6. With increasing Reynolds number, the transition moves upstream, closer to the leading edge. Vortices generated in the transitional area were clearly shorter compared to regimes with Reynolds number $R e_{2 \text { is }}=2.5 \times 10^{5}$.

Moreover some turbulent wedges generated on the blade B can be seen in Figure 7a, b, $\mathrm{c}$ and e. These structures were generated behind the surface defects that were not entirely wiped out from the blade during the surface finishing. It can be seen that smaller defects on the blade surface play more important role in the BL transition if the Reynolds number is higher. 

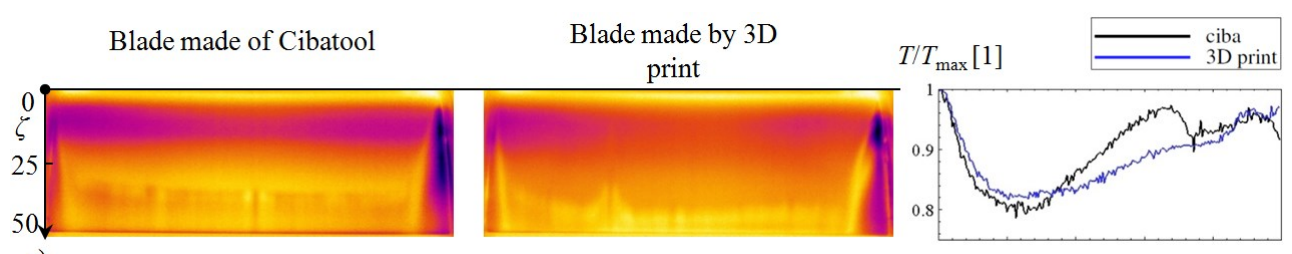

a)
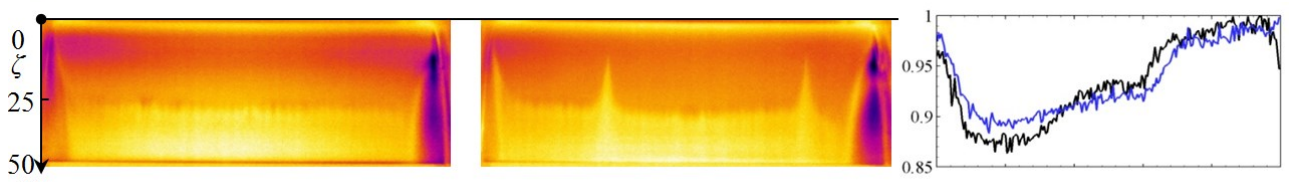

b)
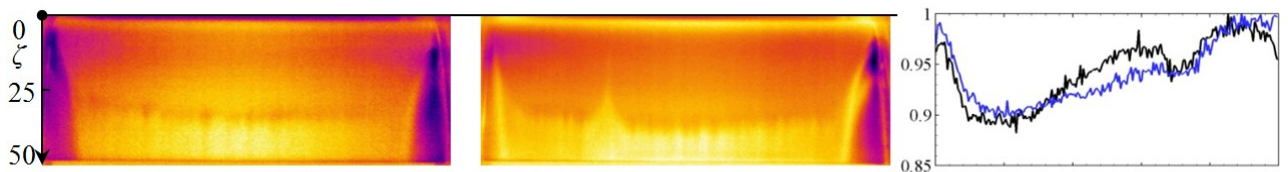

c)
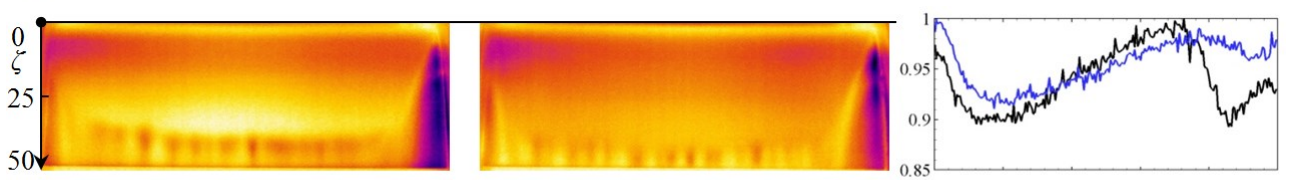

d)
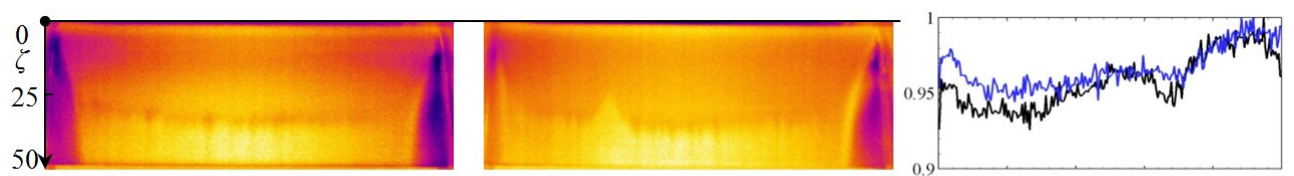

e)
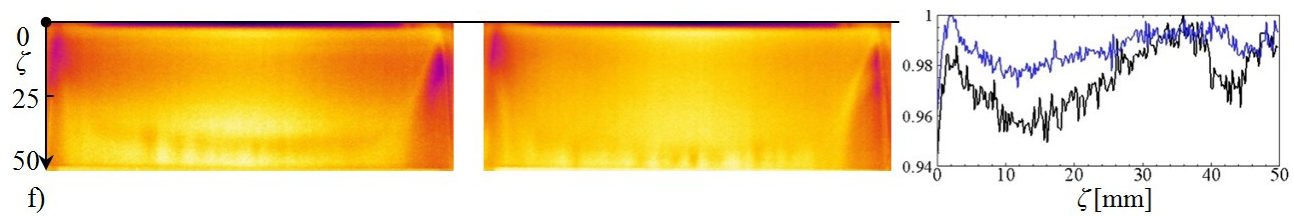

f)

Fig. 7. Visualization of the BL transition using IRT a) $M_{2 \text { is }}=0.8 ; R e_{2 \text { is }}=5 \times 10^{5}$, b) $M_{2 \text { is }}=0.6$; $R e_{2 \text { is }}=7.5 \times 10^{5}, \quad$ c) $M_{2 \text { is }}=0.6 ; \quad R e_{2 \text { is }}=5 \times 10^{5}$, $\left.R e_{2 \text { is }}=5 \times 10^{5}, \mathrm{f}\right) M_{2 \text { is }}=0.4 ; R e_{2 \text { is }}=2.5 \times 10^{5}$.
d) $M_{2 \text { is }}=0.6 ; \quad R e_{2 \text { is }}=2.5 \times 10^{5}$,
e) $M_{2 \text { is }}=0.4$;

\subsubsection{Transition on heated blade $B$}

In order to get higher differences in temperatures of the medium and the blade surface the experiment with heated blade was performed. For this experiment the blade B (manufactured by 3D printing) (Figure 3) was used.

Comparison of heated and unheated blade $\mathrm{B}$ is shown in Figure 8 for Mach number $M_{2 \text { is }}=0.4$ and Reynolds numbers $R e_{2 \text { is }}=(2.5$ and 5$) \times 10^{5}$. Blade was heated up before the visualization using hot air as was described in 2.4. During the visualization itself, heating was turned off, because the temperature distribution was not uniform across the whole blade and before the picture capturing some time was needed for the temperature compensation. Reference surface temperatures $T_{\text {sref }}$ during the measurements are listed in Table 1. 
The heating of the blade had not significant effect on the BL transition as can be seen. The heating of the blade increases the temperature resolution; however, the temperature gradients may affect the evaluation of the blade transition. Areas where the blade was heated more than the rest of the blade can be seen in both figures, moreover in Figure 8b, border of the cavity in the blade can be seen, because the air in it was already colder. Vortex structure behind a surface defect was generated when the Reynolds number was $R e_{2 \text { is }}=5 \times 10^{5}$ (Fig. a) in both investigated cases (heated and unheated blade).

Unheated blade

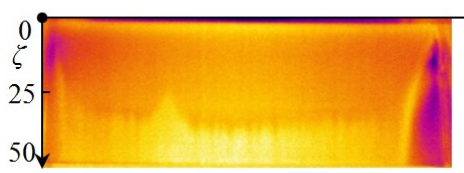

a)

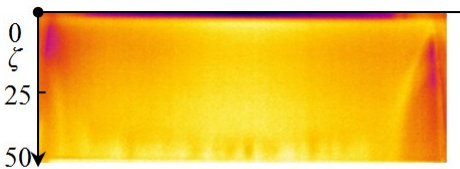

b)
Heated blade
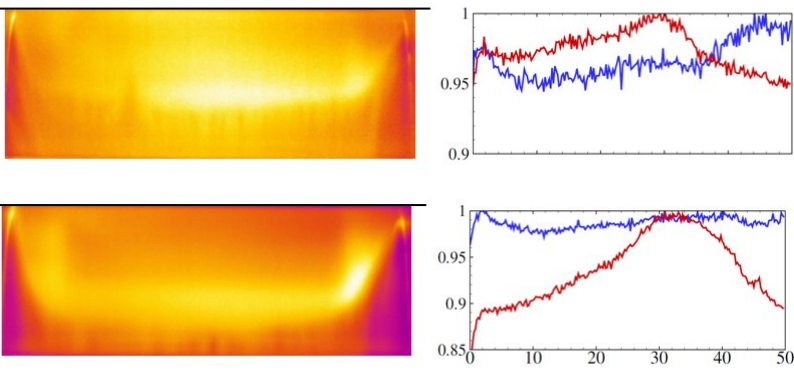

$\zeta\lfloor\mathrm{mm}\rfloor$

Fig. 8. Comparison of the heated and unheated blade B a) $M_{2 \text { is }}=0.4, R e_{2 \text { is }}=5 \times 10^{5}$, b) $M_{2 \text { is }}=0.4$, $R e_{2 \text { is }}=2.5 \times 10^{5}$.

\subsection{Results comparison}

Comparison of the results from both used methods is in Table 2, where the positions $\zeta$ of transitions are summarized. Coordinates $s / c$ used for the description of HFA position was transformed to the system $(\zeta, \xi)$ for better clarity. There is a good agreement of the results obtained by the IRT visualization and by the HFA. Position of the transition obtained from the HFA measurements is evaluated with accuracy of $\zeta= \pm 3.5 \mathrm{~mm}$ because of the distances between individual sensors. Position of the transition obtained from the IRT is evaluated with accuracy of $\zeta= \pm 4 \mathrm{~mm}$, because the transitions were evaluated along the one line positioned in the middle of the blade, but as can be seen from the visualization above, the positions of the transitions were not same along the blade height (value of \pm 4 millimetres was the worst case from all).

Table 2. Results comparison.

\begin{tabular}{|c|c|c|c|c|c|c|c|c|c|}
\hline & & \multicolumn{2}{|c|}{ Blade A } & \multicolumn{2}{c|}{$\begin{array}{c}\text { Blade B } \\
\text { unheated }\end{array}$} & \multicolumn{2}{c|}{$\begin{array}{c}\text { Blade B } \\
\text { heated }\end{array}$} & \multicolumn{2}{c|}{ HFA } \\
\hline $\begin{array}{c}\mathrm{M}_{2 \text { is }} \\
{[1]}\end{array}$ & $\begin{array}{c}\mathrm{Re}_{2 \text { is }} \\
{[1]}\end{array}$ & $\begin{array}{c}\zeta \\
{[\mathrm{mm}]}\end{array}$ & $\begin{array}{c}\mathrm{s} / \mathrm{c} \\
{[1]}\end{array}$ & $\begin{array}{c}\zeta \\
{[\mathrm{mm}]}\end{array}$ & $\begin{array}{c}\mathrm{s} / \mathrm{c} \\
{[1]}\end{array}$ & $\begin{array}{c}\zeta \\
{[\mathrm{mm}]}\end{array}$ & $\begin{array}{c}\mathrm{s} / \mathrm{c} \\
{[1]}\end{array}$ & $\begin{array}{c}\zeta \\
{[\mathrm{mm}]}\end{array}$ & $\begin{array}{c}\mathrm{s} / \mathrm{c} \\
{[1]}\end{array}$ \\
\hline 0.8 & $5.0 \times 10^{5}$ & 34 & 1.011 & 38 & 1.088 & - & - & 38 & 1.088 \\
\hline 0.8 & $2.5 \times 10^{5}$ & - & - & - & - & - & - & 44 & 1.209 \\
\hline 0.6 & $7.5 \times 10^{5}$ & 30 & 0.933 & 30 & 0,933 & - & - & - & - \\
\hline 0.6 & $5.0 \times 10^{5}$ & 32 & 0.972 & 36 & 1.050 & - & - & 35 & 1.030 \\
\hline 0.6 & $2.5 \times 10^{5}$ & 42 & 1.165 & 45 & 1.223 & - & - & 42 & 1.165 \\
\hline 0.4 & $5.0 \times 10^{5}$ & 32 & 0.972 & 36 & 1.050 & 34 & 1.011 & 38 & 1.088 \\
\hline 0.4 & $2.5 \times 10^{5}$ & 40 & 1.127 & 43 & 1.184 & 43 & 1.184 & 42 & 1.165 \\
\hline
\end{tabular}




\section{Conclusions}

The transition of BL on the prismatic blades was determined by IRT for several regimes characterized by Mach and Reynolds numbers. Results were compared to the HFA measurements performed earlier in our laboratory and good agreement was found. The transitions were detected as a rapid change in the temperature of the blade surface, the difference of about $1 \mathrm{~K}$ in temperature between the medium and blade surface was found sufficient. Locations of the transitions were dependent on the value of the Reynolds number $R e_{2 \text { is }}$ (with increasing $R e_{2 \text { is }}$ transition moved toward the leading edge of the blade), no significant influence of Mach number on this phenomenon was observed, what is in accordance with the results made with HFA. The results could be used to verify numerical models. Moreover the corner vortices on the blades can be also detected by IRT. It can be used for the future comparison with measurements performed by multi hole pressure probe behind the cascade, where the kinetic energy losses are usually investigated.

For the future work in this field, some kind of heating or cooling of the bluff body can be recommended, because of better resolution of IRT; however it was shown that technique used during these experiments will need some development.

\section{References}

1. R. Narasimha, Prog. Aerospace Sci., 22, (1985)

2. R.E. Mayle, J. Turbomach. 113, (1991)

3. P. Straka, J. Příhoda, Proc. Conf., Exp. Fluid Mechanics, TU Liberec, (2010)

4. P. Straka, J. Př́ihoda, P. Šafařík, Proc. Conf., App. Exp. Num. Meth. Fluid Mech., Žilina, (2012)

5. F.R. Menter, R.B. Langtry, S.R. Likki, Y.B. Suzen, P.G. Huang, S.A. Völker, J. Turbomach. 128, (2006)

6. Y.B. Suzen, P.G. Huang, J. Fluid Eng. 122, (2000)

7. J. Steelant, E. Dick, Int. J. Num. Methods Fluids 23, (1996)

8. C. Tropea, A.L. Yarin, J.F. Foss, Handbook of experimental fluid mechanics (2007)

9. R.A. Gomes, et al., J. Turbomachinery 137, (2015)

10. P. Griffin, M.R. Davies, $16^{\text {th }}$ Symp. Meas. Tech. Trans. Sup. Flow in Casc. Turb., Cambridge, (2002)

11. S. Zuccher, W.S. Saric, Exp. Fluids 44, 1 (2008)

12. Y. Yokokawa, Trans. Jap. Soc. Aero. Space Sci. 48, 161 (2005)

13. L.A. Joseph, A. Borgoltz, W. Devenport, Exp. Fluids 57, 5 (2016)

14. L. De Luca, G.M. Carlomagno, G. Buresti, Exp. Fluids 9, 3 (1990)

15. J.A. Patorski, G.S. Bauer, J. Theor. App. Mech. 39, 2 (2001)

16. P. Baek, P. Fuglsang, Eur. Wind Energy Conference, (2009)

17. M. Raffel, Ch.B. Merz, T. Schwermer, K. Richter, Exp. Fluids 56, 2 (2015)

18. T. Jelínek, Exp. Fluid Mech. conf. proc., (2017)

19. Guide to the Expression of Uncertainty in Measurement, JCGM 100:2008, (1995)

20. J. Michálek, VZLU technical report R-6541, (2016) 